\title{
COMMUNICATIONS IN CONTEMPORARY MATHEMATICS
}

\section{Author Index Volume 2 (2000)}

Attouch, H., Goudou, X. \&

Redont, P.,

The heavy ball with friction method, I. The continuous dynamical system: Global exploration of the local minima of a real-valued function by asymptotic analysis of a dissipative dynamical system

Braides, A.,

Non-local variational limits of discrete systems

Cao, J.,

Cheeger isoperimetric constants of Gromov-hyperbolic spaces with quasi-poles

Dasbach, O. T.,

On the combinatorial structure of primitive Vassiliev invariants, III - A lower bound

de Figueiredo, D. G., Goncalves, J. V. \& Miyagaki, O. H.,

On a class of quasilinear elliptic problems involving critical exponents

Etnyre, J. B.,

Tight contact structures on lens spaces

Fang, F. \& Rong, X.,

Fixed point free circle actions and finiteness theorems

García Azorero, J. P., Peral Alonso, I. \& Manfredi, J. J.,

Sobolev versus Hölder local minimizers and global multiplicity for some quasilinear elliptic equations

Goncalves, J. V., see de Figueiredo

Goudou, X., see Attouch

Hang, F.-B. \& Lin, F.-H., A remark on the Jacobians

He, H. (Jacob Livingstone),

Theta correspondence I Semistable range: Construction and irreducibility

Hind, R.,

Holomorphic filling of $\mathrm{R} P^{3}$

Huang, Y.-Z. \& Zhao, W.,

Semi-infinite forms and topological vertex operator algebras
1(2000)1

2(2000)285

4(2000)511

$\mathbf{4}(2000) 579$

1(2000)47

4(2000)559

1(2000)75

3(2000)385

1(2000)47

1(2000)1

1(2000)35

2(2000)255

3(2000)349

2(2000)191
Jiang, B. \& Wang, S.,

Achirality and planarity

Lim, Y.,

Seiberg-Witten moduli spaces for 3-manifolds with cylindrical-end $T^{2} \times R^{+}$

Lin, F.-H., see Hang

Liu, G. \& Tian, G., Weinstein conjecture and GWinvariants

Lupo, D. \& Payne, K. R., On the maximum principle for generalized solutions to the Tricomi problem

Manfredi, J. J., see García Azorero

Mawhin, J., Rebelo, C. \&

Zanolin, F.,

Continuation theorems for Ambrosetti-Prodi type periodic problems

Miyagaki, O. H., see de Figueiredo

Ortega, R. \& Tarallo, M.,

Degenerate equations of pendulum-type

Payne, K. R., see Lupo

Peltonen, K. Contact structures on $S^{3}$ and Seifert fibrations

Peral Alonso, I., see García Azorero

Planchon, F.,

On the cauchy problem in Besov spaces for a non-linear Schrödinger equation

Rebelo, C.,

1(2000)87

see Mawhin

Redont, P., see Attouch

Rong, X., see Fang

Salur, S., Deformations of special Lagrangian submanifolds

Shafrir, I.,

3(2000)299

$4(2000) 461$

1(2000)35

4(2000)405

$4(2000) 535$

3(2000)385

1(2000)87

1(2000)47

2(2000)127

$4(2000) 535$

1(2000)61

3(2000)385

2(2000)243

1(2000)1

1(2000)75

3(2000)365

2(2000)151

Asymptotic behaviour of minimizing sequences for Hardy's inequality 
Suzuki, T., A note on the stability of stationary solutions to a system of chemotaxis

Tarallo, M., see Ortega

Tian, G., see Liu

Wang, S., see Jiang

$\mathbf{3}(2000) 373$
$\mathbf{2}(2000) 127$
$\mathbf{4}(2000) 405$
$\mathbf{3}(2000) 299$

$\mathrm{Xu}, \mathrm{F}$, Jones-Wassermann subfactors for disconnected intervals

Zanolin, F., see Mawhin

Zhao, W., see Huang
3(2000)307

1(2000)87

2(2000)191 